\title{
Association Between Soluble CD40L with Thrombosis Occurrence and JAK2 V617F Mutation in Essential Thrombocythemia
}

\author{
Wafaa S. Mohammed ${ }^{1}$, Wael A. Abbas ${ }^{2,}$, , Ola A. Afifi ${ }^{1}$, Ahmad F. Thabet ${ }^{2}$ \\ ${ }^{1}$ Clinical Pathology Department, Faculty of Medicine, Assiut University, Assiut, Egypt \\ ${ }^{2}$ Internal Medicine Department, Faculty of Medicine, Assiut University, Assiut, Egypt \\ Email address: \\ Drwaelabbas@yahoo.com(W.A. Abbas),Wafaa_salah_elsaid@hotmail.com(W. S. Mohammed), Olaafifi18@yahoo.com (O. A. Afifi), \\ Drahmedfarag2005@yahoo.com (A. F. Thabet) \\ ${ }^{*}$ Corresponding author
}

\section{To cite this article:}

Wafaa S. Mohammed, Wael A. Abbas, Ola A. Afifi, Ahmad F. Thabet. Association Between Soluble CD40L with Thrombosis Occurrence and JAK2 V617F Mutation in Essential Thrombocythemia. American Journal of Internal Medicine. Vol. 4, No. 4, 2016, pp. 60-65. doi: 10.11648/j.ajim.20160404.11

Received: May 2, 2016; Accepted: May 21, 2016; Published: June 4, 2016

\begin{abstract}
Thrombo-haemorrhagic events are the main cause of mortality in essential thrombocythemia (ET). The aim of this study was to measure soluble CD40 ligand (sCD40L) in the plasma of healthy individuals and in patients with an elevated platelet count and investigate the association of SCD40L with thrombosis in ET patients and their JAK2 V617F mutation. The plasma levels of sCD40L was measured in 75 patients. 35 patients diagnosed as ET, 25 patients diagnosed as reactive thrombocytosis (RT), 15 patients with low platelet count and 15 healthy subjects acted as the control group. 35 ET patients were assessed for JAK2 V617F status by utilizing a JAK2 V617F specific quenching probe. ET patients had the highest levels of SCD40L compared to the patients with RT and controls $(225.70 \pm 79.34,160.40 \pm 54.54$ and $83.54 \pm 21.54)$ respectively and a tight correlation was found between the platelet count and sCD40L. Statistical analysis revealed that the JAK2 V617F mutation was associated with significantly increased levels of WBCs $(p<0.04)$ and sCD40L $(p<0.001)$ compared to JAK2 V617F negative patients. There was no significant association between JAK2 V617F mutation and thrombosis, but the level of SCD40L was significantly higher in patients with thrombosis than those without thrombosis $(236.43 \pm 75.93$ vs $184.65 \pm$ 62.31 ) respectively. Based on these findings, the presence of JAK2 mutation may changes the expression of soluble markers of endothelial and platelet activation besides the quantitative and qualitative changes in platelets. Mechanisms leading to thrombosis are more complex and multifactorial.
\end{abstract}

Keywords: Essential Thrombocythemia, sCD40L, JAK2 V617F Mutation, Thrombosis

\section{Introduction}

Essential thrombocythaemia (ET) is a myeloproliferative neoplasm characterized by an increase in the peripheral blood platelet count that is associated with bone marrow megakaryocyte hyperplasia, without associated erythrocytosis or leukoerythroblastosis [1].

According to different reports, $50 \%$ to $60 \%$ of patients with ET have mutation V617F of the Janus Kinase (JAK2) gene [2]. A single $G$ to $T$ somatic point mutation at nucleotide 1,849 in exon 14 of JAK2 results in the substitution of valine with phenylalanine at codon 617 (JAK2 V617F), consequently increasing the tyrosine kinase activity leading to abnormal expansion of myeloid cells [3-5].

It has been determined that the JAK2 V617F mutation occurs at the stem cell level, and is the major molecular mechanism, as well as a potential diagnostic marker for the development of MPNs, including PV and ET [6-8]. Many studies have been performed to evaluate the association of JAK2 V617F with the risk of thrombosis, especially in patients with ET, but the results have been inconclusive [9-12].

CD40L is expressed in activated CD4 $\mathrm{T}$ lymphocytes and platelets and is converted to $\mathrm{sCD} 40 \mathrm{~L}$ via a proteolytic 
process [13]. On platelet activation, P-selectin and CD40 ligand are rapidly translocated to the platelet surface and subsequently cleaved to generate soluble forms fully active biologically, termed soluble P-selectin (sPselectin) and soluble CD40 ligand (sCD40L) [14, 15], both of which promote coagulation by inducing tissue factor (TF) expression on the monocytes and the endothelial cells [16]. Soluble CD40L also causes platelet activation and appears to be required for thrombus formation in vivo [15, 17]. However, the presence of CD40L in platelets makes possible the assumption that circulating $\mathrm{sCD} 40 \mathrm{~L}$ derives from the platelets and that the observed variations of $\mathrm{sCD} 40 \mathrm{~L}$ concentrations in disease states are related to the platelet count. These observations provided the rationale for our study. We asked whether there was a relationship between platelet number and concentration of SCD40L in plasma in healthy individuals and in patients with an elevated platelet count.

Considering that thrombosis is a major cause of morbidity and mortality in patients with ET [18] and a remarkable proportion (50-40\%) of ET patients are reported to be JAK2 V617F negative cases, yet thrombotic complications can also be observed in this group $[19,20]$. So, the identification of a biological marker for high risk patients with ET would be of great clinical utility. Therefore, we investigated the association of sCD40L with thrombosis in ET patients and their JAK2 V617F mutation.

\section{Study Design}

\subsection{Patients and Controls}

The study was carried out at the Department of Internal Medicine, Division of Hematology and Clinical Lab, Assiut University Hospital, Assiut, Egypt. The study group included 75 patients. 35 patients were diagnosed as having ET (11 male $/ 24$ female) with a mean age of $54.62 \pm 7.02$. The diagnostic criteria for ET were those recommended and updated by the World Health Organization (WHO) in 2008 [21]. 25 patients ( 8 male / 17 female) with a mean age of $58.4 \pm 7.53$ were diagnosed as having RT (patients with a platelet count above $450 \times 10^{9} / \mathrm{L}$, without a myeloproliferative disorder, and with an underlying disease likely associated with RT). In each case the platelet count normalized if the acute disease state was no longer present after treatment.

Causes of RT were malignancy (10 patients), infection (8), anemia attributed to iron deficiency (7). 15 patients with low platelet levels $\left(<100 \times 10^{9} / \mathrm{L}\right)(4$ male / 11 female) with a mean age of $52.76 \pm 7.49$ included 2 patients with myelodysplasia, 9 patients with idiopathic thrombocytopenic purpura (ITP), and 4 patients with secondary thrombocytopenia resulting from an autoimmune or lymphoproliferative disorder. Controls were 15 healthy blood donors (5 male/10 female) with a mean age of $45.60 \pm 13.12$ who were sex and age matched with patients. None of the patients with ET or healthy subjects had symptoms of an acute infection or inflammatory diseases.
The study was approved by the local ethics committee and was conducted in accordance with the Declaration of Helsinki. Informed consent was obtained from all patients enrolled into this study.

\subsection{Laboratory Analyses}

Whole blood was routinely collected into tubes containing ethylene diaminetetraacetic acid (Becton Dickinson, Mountain View, CA) and was immediately centrifuged at $3000 \mathrm{~g}$ at $4^{\circ} \mathrm{C}$. Plasma samples were a liquated and stored at $80^{\circ} \mathrm{C}$ until assay. Repeated freezing and thawing process was avoided.

\subsubsection{Routine Hematological Assays}

White blood cell count, hematocrit, hemoglobin and platelet counts were determined by automated methods using a Cell-Dyn 3500 Analyzer (Abbot).

\subsubsection{Soluble CD40L Elisa}

Quantification of sCD40L in plasma was performed with a commercial enzyme-linked immunosorbent assay (ELISA) kit (R\&D Systems, Minneapolis, MN, USA) according to manufacturer's instructions.

\subsubsection{JAK2 V617F Mutation Analysis}

DNA extraction: Genomic DNA was isolated from whole EDTA blood using QIAamp DNA Mini kit (Qiagen, Germany) according to manufacturer's instructions.

Real time quantitative polymerase chain reaction (RTPCR) assay for JAK2 V617F mutation.: using Fast 7500 Applied Biosystems. The presence of the JAK2V617F mutation was investigated as described by Baxter et al [6]. Briefly, $80 \mathrm{ng}$ of DNA from the patients were used to amplify the mutated and unmutated exon 14 of $J A K 2$ in an allele-specific polymerase chain reaction (PCR). PCR products were separated on a $3 \%$ agarose gel, stained with ethidium bromide, and viewed under UV light. A 203 basepair fragment indicates the presence of the $1849 \mathrm{G}>\mathrm{T}$ mutation. A quantitative real-time PCR-based allelic discrimination assay was used to detect the JAK2V617F mutation employing TaqMan real-time technology. Genomic DNA was amplified in a 40-cycle PCR at an annealing temperature of $61^{\circ} \mathrm{C}$. All reactions were carried out in a final volume of $25 \mu \mathrm{L}$ containing $1 \times$ PCR Master Mix (Applied Biosystems), $900 \mathrm{nM}$ of both forward and reverse primers and $100 \mathrm{nM}$ of each probe. A ratio between the $\mathrm{Ct}$ (JAK2 V617F) and $\mathrm{Ct}$ (JAK2 wild-type) was calculated for each sample. cDNA pooled from ten Donors were used as the calibrator sample. The sequences of the primers and probes are listed in table 1.

\subsection{Statistical Analyses}

All statistical analyses were performed using the Statistical Package for Social Sciences (SPSS, version 21.0 for Windows, Chicago, Ill, USA). Data were expressed as mean \pm standard deviation (SD). A "p"value $<0.05$ was accepted as significant. Mann-whitney $U$ test was used to compare 
continuous variables and Chi-square to compare categorical was applied and Pearson coefficient of correlation (r) was variables between the groups. Pearson correlation analysis used to show the relationship between the variables.

Table 1. Sequences of the real-time primers and probes for JAK2.

\begin{tabular}{lll}
\hline 5'forword primer & 5'reverse primer & 5'probe \\
\hline \multirow{2}{*}{ AAGCTTTCTCACAAGCATTTGGTTT } & AGAAAGGCATTAGAAAGCCTGTAGTT & MGB \\
& & JAK2(A) FAM 5'-TCCACAGAA ACATAC \\
& JAK2(C) VIC 5'-CTCCACAGACACATAC \\
\hline
\end{tabular}

\section{Results}

Levels of sCD40L were measured by ELISA in the plasma of 75 patients and 15 healthy subjects act as the control group.

All patients with high platelet counts (RT and ET) had significant raised levels of SCD40L with a mean value of $(160.40 \pm 54.54$ and $225.7 \pm 79.34$, respectively) when compared with controls $(83.54 \pm 21.54)$ and patients with low platelet counts (23.40 \pm 4.33$)$. Highest levels of sCD40L were seen within the ET population figure 1. A positive correlation between $\mathrm{SCD} 40 \mathrm{~L}$ and platelet counts was observed $(\mathrm{r}=0.889$, $\mathrm{p}<0.000$ ) figure 2 .

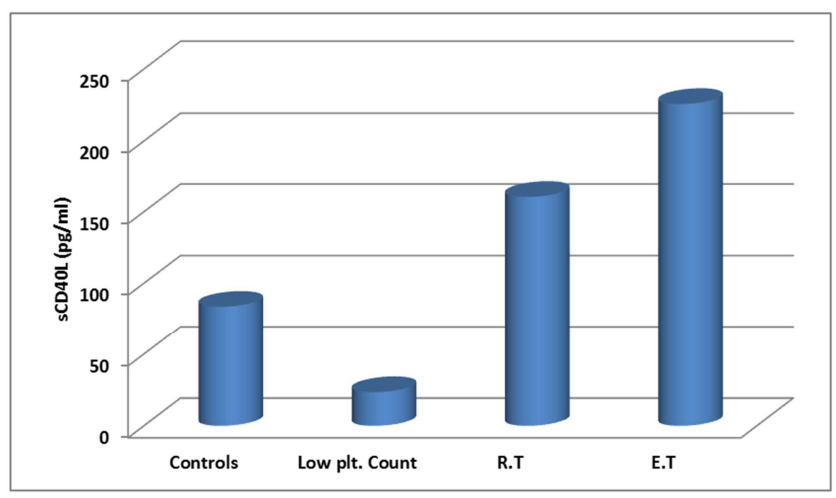

Figure 1. Levels of SCD40L in the patients and healthy controls.

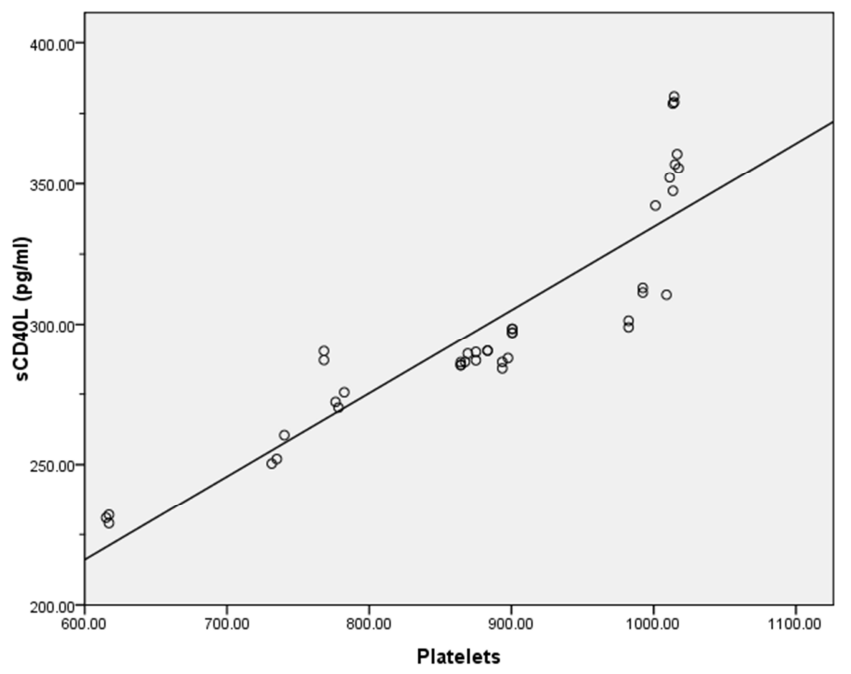

Figure 2. Correlations between the platelet count $\left(* 10^{9} / \mathrm{L}\right) \&$ the plasma concentrations of SCD40L $(r=0.889, P<0.000)$.

At the time of study, ET patients received one or more of the following therapies: anti-platelet agents (aspirin) and myelosuppressive drugs (e.g: hydroxyurea). Patients with ET had significantly higher white blood cells $(p<0.02 *)$ and platelet counts $\left(\mathrm{p}<0.000^{* * *}\right)$ compared to healthy controls.

The main demographic and clinical data of ET patients and healthy controls were summarized in table 2 .

Table 2. Demographic and clinical data of controls and ET patients.

\begin{tabular}{|c|c|c|c|}
\hline Item & ET $n=35$ & Control $n=15$ & p-value \\
\hline \multicolumn{4}{|l|}{ 1-Age "years" } \\
\hline mean \pm SD & $54.62 \pm 7.02$ & $45.60 \pm 13.12$ & \multirow{3}{*}{$\begin{array}{l}\mathrm{p}=0.483 \\
\text { (n.s) }\end{array}$} \\
\hline (min-max) & $(48.0-70.0)$ & $(31.0-69.0)$ & \\
\hline 2-Sex: & & & \\
\hline \multirow{2}{*}{$\mathrm{M} / \mathrm{F}$} & $11(31.42 \%)$ & $5(33.33 \%)$ & \multirow{3}{*}{$\begin{array}{l}\mathrm{p}=0.582 \\
\text { (n.s) } \\
\mathrm{P}=0.667 \\
\text { (n.s) }\end{array}$} \\
\hline & $/ 24(68.57 \%)$ & $/ 10(66.67 \%)$ & \\
\hline 3-Hct"\%" & $41.32 \pm 7.77$ & $40.83 \pm 8.56$ & \\
\hline $\begin{array}{l}\text { 4-WBCs count } \\
\left(* 10^{9} / \mathrm{L}\right)\end{array}$ & $8.23 \pm 2.56$ & $6.43 \pm 1.89$ & $\mathrm{p}<0.02 *$ \\
\hline $\begin{array}{l}\text { 5-Platelets count } \\
\left(* 10^{9} / \mathrm{L}\right)\end{array}$ & $792.54 \pm 275.53$ & $242.66 \pm 79.32$ & $\mathrm{p}<0.000 * * *$ \\
\hline 6-Splenomegaly & $8(22.85 \%)$ & -- & -- \\
\hline $\begin{array}{l}\text { 7-History of } \\
\text { DVT }\end{array}$ & $13(37.14 \%)$ & -- & -- \\
\hline $\begin{array}{l}\text { 8-History of } \\
\text { bleeding }\end{array}$ & -- & -- & -- \\
\hline
\end{tabular}

Data presented as mean \pm SD. $p<0.05$ is significant

Table 3. Clinical features in 35 patients with ET according to JAK2 mutation.

\begin{tabular}{|c|c|c|c|}
\hline \multirow{2}{*}{ Item } & Jak2 V617F +ve & Jak2 V617F -ve & \multirow{2}{*}{ p-value } \\
\hline & $N=19$ & $N=16$ & \\
\hline $\begin{array}{l}\text { 1-Age "years" } \\
\text { mean } \pm \text { SD } \\
\text { 2-Sex: }\end{array}$ & $56.25 \pm 12.65$ & $52.43 \pm 15.43$ & $\mathrm{P}=0.639 \mathrm{n} . \mathrm{s}$ \\
\hline $\mathrm{M} / \mathrm{F}$ & $\begin{array}{l}6(31.57 \%) \\
/ 13(68.42 \%)\end{array}$ & $\begin{array}{l}5(31.25 \%) \\
/ 11(68.75 \%)\end{array}$ & $\mathrm{P}=0.685 \mathrm{n} . \mathrm{s}$ \\
\hline 3-Hct"\%" & $41.76 \pm 9.38$ & $40.55 \pm 9.16$ & $\mathrm{P}=0.582 \mathrm{n} . \mathrm{s}$ \\
\hline $\begin{array}{l}\text { 4- WBCs count } \\
\left(* 10^{9} / \mathrm{L}\right)\end{array}$ & $9.82 \pm 3.04$ & $7.5 \pm 2.38$ & $\mathrm{P}<0.04 *$ \\
\hline $\begin{array}{l}\text { 5- Platelets count } \\
\left(* 10^{9} / \mathrm{L}\right)\end{array}$ & $815.24 \pm 201.21$ & $850.42 \pm 210.38$ & $\mathrm{P}=0.758 \mathrm{n} . \mathrm{s}$ \\
\hline 6-Splenomegaly & $5(26.31 \%)$ & $3(18.75 \%)$ & $\mathrm{P}=0.582 \mathrm{n} . \mathrm{s}$ \\
\hline $\begin{array}{l}\text { 7-History of } \\
\text { thrombosis }\end{array}$ & $7(36.84 \%)$ & $6(37.50 \%)$ & $\mathrm{P}=0.527 \mathrm{n} . \mathrm{s}$ \\
\hline 8 -sCD40L $(\mathrm{pg} / \mathrm{ml})$ & $245.23 \pm 115.43$ & $171.22 \pm 74.65$ & $\mathrm{P}<0.001^{* *}$ \\
\hline
\end{tabular}

Data presented as mean \pm SD. $\mathrm{p}<0.05$ is significant

Detectable amounts of JAK2 V617F DNA in whole blood were found in 19 out of 35 patients with ET (54.28\%) figure 3,4 . No mutation was identified in the controls or in any patients with RT, demonstrating the high specificity of the JAK2 V617F mutation in classical MPNs. As summarized in 
table 3, the JAK2 V617F positive patients had significantly higher mean $\mathrm{WBC}$ and $\mathrm{SCD} 40 \mathrm{~L}$ than those without the mutation $\left(\mathrm{p}<0.04^{*}\right.$ and $\mathrm{p}<0.001^{* *}$, respectively). Thrombotic events were observed in $36.84 \%$ of patients with JAK2 V617F compared with $37.50 \%$ of patients without JAK2 V617F.

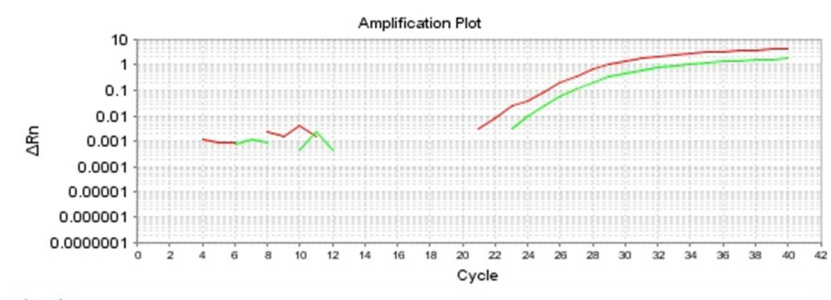

JAK2-Wild type $\square$ JAK2-V617F Mutant

Figure 3. Patient positive for JAK2 mutation expression.

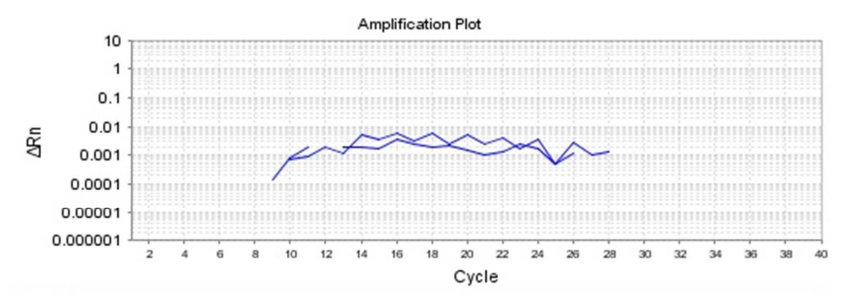

JAK2

Figure 4. Patient negative for JAK2 mutation expression.

We found no statistical difference in age, sex, hematocrit levels, splenomegaly rate, or thrombosis occurrence according to JAK2 mutation status. Patients with a history of thrombosis had significantly higher sCD40L levels than those without thrombosis $(236.43 \pm 75.93$ vs $184.65 \pm 62.31$, p $<0.001 *)$ and controls $(83.54 \pm 21.54, \mathrm{p}<0.001 * *)$ figure 5 .

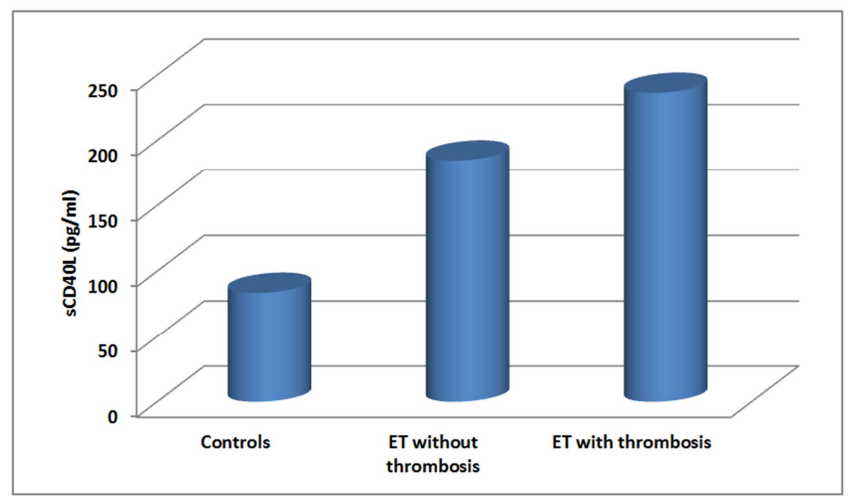

Figure 5. Concentration of $s C D 40 L$ in matched healthy controls and ET patients without or with thrombosis.

\section{Discussion}

CD40 ligand (CD40L) is a type II transmembrane protein that is expressed on activated CD4+ T lymphocytes and can be released as a soluble form $(\mathrm{sCD} 40 \mathrm{~L})$ by an ill-defined proteolytic process. Although it is known that CD40L is expressed over the surface of activated platelets and that this is followed by a subsequent release of $\mathrm{sCD} 40 \mathrm{~L}$ from platelets, sCD40L levels may vary according to platelet count and/or the disease state $[13,22]$.

In our study, sCD40L levels were significantly higher in all patients with high platelet counts compared with controls. Highest levels were seen within the ET population and this higher level showed parallelism with platelet counts, which was consistent with the previous studies [23, 24]. These results revealed that there was a significant correlation between platelet count and SCD40L in patients with ET and that levels were also increased in reactive thrombocytosis, with sCD40L levels being increased when the platelet count is high. The fact that sCD40 levels correlate with platelet count shows that $\mathrm{SCD} 40$ is found largely in platelets though expressed in many other cells. A correlation between plateletassociated CD40 and soluble CD40 levels and increased levels of $\mathrm{sCD} 40 \mathrm{~L}$, as a marker of platelet regeneration, where there is increase megakaryopoiesis, has also been reported.

Essential Thrombocythemia (ET) is characterized by persistently elevated platelet counts in the context of a normal red cell mass [25]. Several diagnostic algorithms for this disorder have been proposed. However, irrespective of the criteria employed for diagnosis, patients with ET comprise a heterogeneous group. Heterogeneity is manifested both by the variation in clinical course observed, and by the variable presence of cellular and molecular markers [26]. JAK2 V617F mutation has been recognized as a possible thrombotic risk factor in ET. Its role is probably due to an increased myeloid proliferation and white blood cells (WBCs) activation [27].

In the present study, screening of JAK2 V617F was performed in 35 patients with ET according to the WHO diagnostic criteria $[21,28]$, we found a $54.28 \%$ incidence of the JAK2 V617F mutation among our ET patients, a finding in agreement with different reports that described point mutation in the JAK2 kinase (JAK2 V617F) in about 30-70\% of ET patients [2, 29-31].

In contrast to other investigators [27, 32-34], a nonsignificant association between the JAK2 V617F mutation and the thrombotic events was detected in current study group. Although the JAK2 V617F mutation was associated with significantly increased levels of WBC counts but its direct effect on the thrombotic events did not prove its suggested predictive potential. However, the present study results indirectly support the concept that the JAK2V617F mutation contributes to intrinsic changes in both megakaryocyte and platelet biology beyond the increase in cell numbers [35-37]. The results of another single center analyses also revealed that the JAK2 V617F (-) cases did not differ significantly from the JAK2 V617F $(+)$ cases in the incidence of thrombosis [38-40]. The number of involved patients might be determinative in view of clear evaluation of the predictive value of the JAK2 V617F mutation in thrombosis [41, 42]. Neither hematocrit levels nor platelet counts showed significant differences in patients with JAK2 $\mathrm{V} 617 \mathrm{~F}$ mutation than in those without, as reported in 
previous studies [12, 34, 43].

The role of $\mathrm{sCD} 40 \mathrm{~L}$ in the thrombosis of ET was assessed in this study, as well as its relationship with the patients' JAK2 mutation status. Similar to this study, previous studies reported elevated SCD40L levels in ET patients with thrombosis $[23,44]$. In addition, sCD40L induces platelet $\mathrm{P}$ selectin expression, aggregation, leukocyte activation, platelet-leukocyte conjugation, and platelet release of reactive oxygen intermediates $[15,16,45,46]$. Our findings suggest that $\mathrm{SCD} 40 \mathrm{~L}$, which are released from the activated platelets, could play a role in the pathogenesis of ET thrombosis.

Our study confirmed the previous finding of increased plasma concentrations of SCD40L in ET patients with the JAK2 mutation [47]. Furthermore, these data analyzed the relationship between JAK2 mutation status and this activation marker according to the proportion of mutant alleles. A JAK2 mutant allele dosage effect could be demonstrated on sP-selectin, sCD40L, TF, and VWF: Ag levels, with the highest plasma levels being found in patients with more than $12 \%$ JAK2 V617F mutant alleles, supporting the role for $\mathrm{sCD} 40 \mathrm{~L}$ in thrombosis of ET as well as the association between JAK2 V617F allele burden and soluble markers of platelet and endothelial activation.

\section{Conclusion}

In conclusion, the impact of JAK2 V617F mutation in the prediction of subsequent thrombotic events could not be proven in this study. Our results indirectly support the concept that, besides the quantitative and qualitative changes in the platelets, the mechanism leading to thrombosis is more complex with multifactorial contributions.

\section{References}

[1] Landolfi R, Cipriani MC, Novarese L (2006). Thrombosis and bleeding in polycythemia vera and essential thrombocythemia: pathogenetic mechanisms and prevention. Best Pract Res Clin Haematol; 19: 617-33".

[2] Tefferi A, Gilliland DJ (2005). The JAK2V617F tyrosine kinase mutation in myeloproliferative disorders: status report and immediate implications for disease classification and diagnosis. Mayo Clin Proc; 80: 947-58".

[3] Kralovics R, Passamonti F, Buser AS, et al., (2005). A gain-of-function mutation of JAK2 in myeloproliferative disorders. N Engl J Med 352: 1779-1790".

[4] Levine RL, Wadleigh M, Cools J, et al., (2005). Activating mutation in the tyrosine kinase JAK2 in polycythemia vera, essential thrombocythemia and myeloid metaplasia with myelofibrosis. Cancer Cell; 7: 387-397".

[5] James C, Ugo V, Le Couédic JP, et al., (2005). A unique clonal JAK2 mutation leading to constitutive signaling causes polycythaemia vera. Nature; 434: 1144-1148".

[6] Baxter EJ, Scottl M, Campbell PJ, et al., (2005). Cancer Genome Project: Acquired mutation of the tyrosine kinase
JAK2 in human myeloproliferative disorders. Lancet; 365: 1054-1061".

[7] Scott LM, Tong W, Levine RL, et al., (2007). JAK2 exon 12 mutations in polycythemia vera and idiopathic erythrocytosis. N Engl J Med; 356: 459-468".

[8] Levine RL, Belisle C, Wadleigh M, et al., (2006). $\mathrm{X}$-inactivation-based clonality analysis and quantitative JAK2V617F assessment reveal a strong association between clonality and JAK2V617F in PV but not ET/MMM, and identifies a subset of JAK2V617F-negative ET and MMM patients with clonal hematopoiesis. Blood; 107: 4139-4141".

[9] Lussana F, Caberlon S, Pagani C, et al., (2009). Association of V617F Jak2 mutation with the risk of thrombosis among patients with essential thrombocythemia or idiopathic myelofibrosis: Asystemic review. Thrombosis Research, 124: 409-417".

[10] Linnemann B, Kraft C, Roskos M, et al (2012). Inferior vena cava thrombosis and its relationship with the JAK2V617F mutation and chronic myeloproliferative disease. Thrombosis Research, 129: 720-724".

[11] Austin SK, Lambert JR (2008). The JAK2V617F mutation and thrombosis. Br. J. Haematology, 143: 307-320".

[12] Barbui T, Finazzi G, Falanga A (2013). Myeloproliferative neoplasms and thrombosis. Blood, 122: 2176-2184".

[13] Wykes M, Poudrier J, Lindstedt R, et al., (1998). Regulation of cytoplasmic, surface and soluble forms of CD40 ligand in mouse B cells. Eur J Immunol; 28: 548-59".

[14] Berger G, Hartwell DW, Wagner DD (1998). P-selectin and platelet clearance. Blood; 92: 4446-4452".

[15] Inwald DP, McDowall A, Peters MJ, et al., (2003). CD40 is constitutively expressed on platelets and provides a novel mechanism for platelet activation. Circ Res; 92: 1041-1048".

[16] Lindmark E, Tenno T, Seigbahn A (2000). Role of P-selectin and CD40 ligand in the induction of monocyte tissue factor expression. Arterioscler Thromb Vasc Biol; 20: 2322-2328.

[17] Prasad KSS, Andre P, He M, et al., (2003). Soluble CD40 ligand induces b3 integrin tyrosine phosphorylation and triggers platelet activation by outside-in signaling. Proc Natl Acad Sci USA; 100: 12367-12371".

[18] Cervantes F, Passamonti F, Barosi G (2008). Life expectancy and prognostic factors in the classic BCR/ABL-negative myeloproliferative disorders. Leukemia; 22: 905-914".

[19] Matsumura I, Horikawa Y, Kanakura Y (1999). Functional roles of thrombopoietin-c-mpl system in essential thrombocythemia. Leuk Lymphoma, 32(3-4): 351-358".

[20] Craig S, Kitchens BAK, Craig M, et al., (2013). Consultative hemostasis and thrombosis. Elsevier Health Sciences(ed)".

[21] Wadleigh M, Tefferi A (2010). Classification and diagnosis of myeloproliferative neoplasms according to 2008 World Health Organization criteria. Int J Hematol; 91: 174-179".

[22] Aukrust P, Muller F, Ueland T, et al (1999). Enhanced levels of soluble and membrane-bound CD40 ligand in patients with unstable angina. Possible reflection of $\mathrm{T}$ lymphocyte and platelet involvement in the pathogenesis of acute coronary syndromes. Circulation; 100: 614-20". 
[23] Viallard JF, Solanilla A, Gauthier B et al., (2002). Increased soluble and platelet-associated CD40 ligand in essential thrombocythemia and reactive thrombocytosis. Blood; 99: 2612-4".

[24] Bilgir F, Bilgir O, Kebapcilar L, et al., (2012). Soluble CD40 ligand, high sensitive $\mathrm{C}$-reactive protein and fetuin-A levels in patients with essential thrombocythemia. Transfusion and Apheresis Science; 46: 67-71".

[25] Tefferi A, Vardiman JW (2008). Classification and diagnosis of myeloproliferative neoplasms: the 2008 World Health Organization criteria and point-of-care diagnostic algorithms. Leukemia; 22(1): 14-22".

[26] Tefferi A (2012). Polycythemia vera and essential thrombocythemia: 2012 update on diagnosis, risk stratification, and management. Am J Hematol; 87(3): 285293".

[27] Patriarca A, Pompetti F, Malizia R et al., (2010). Is the absence of JAK2 V617F mutation a risk factor for bleeding in essential thrombocythemia? An analysis of 106 patients. Blood Transfusion; 8: 21-27".

[28] Swerdlow SH, Campo E, Harris NL, et al., (2008). WHO classification of tumors of haematopoietic and lymphoid tissues. WHO press; 76-86".

[29] Campbell PJ, Scott LM, Buck G, et al., (2005). Definition of subtypes of essential thrombocythaemia and relation to polycythaemia vera based on JAK2V617F mutation status: a prospective study. Lancet: 366; 1945-53".

[30] Antonioli E, Guglielmelli P, Poli G etal., (2008). Influence of JAK2V617F allele burden on phenotype in essential thrombocythemia. Haematologica; 93: 41".

[31] Zhang SP, Li H and Lai RS (2015). Detection of JAK2 V617F mutation increases the diagnosis of myeloproliferative neoplasms. ONCOLOGY LETTERS; 9: 735-738".

[32] Kittur J, Knudson RA, Lasho TL, et al., (2007). Clinical correlates of JAK2 V617F allele burden in essential thrombocythemia. Cancer; 109: 2279-2284".

[33] Carobbio A, Antonioli E, Guglielmelli P et al., (2008). Leukocytosis and risk stratification assessment in essential thrombocythemia. J Clin Oncol; 26: 2732-2736".

[34] Takata Y, Seki R, Kanajii T, et al., (2013). Association between thromboembolic events and the JAK2 V617F mutation in myeloproliferative neoplasms. kurume Medical Journal; 60: 89-97".

[35] Hsiao HH, Yang MY, Liu YC, et al., (2007). The association of JAK2V617F mutation and leukocytosis with thrombotic events in essential thrombocythemia. Exp Hematol; 35(11): $1704-1707$
[36] Vannucchi AM, Barbui $T$ (2007). Thrombocytosis and thrombosis. Hematology Am Soc Hematol Educ Program; 363-370".

[37] Hobbs CM, Manning H, Bennett C, et al., (2013). JAK2V617F leads to intrinsic changes in platelet formation and reactivity in a knock-in mouse model of essential thrombocythemia. Blood".

[38] Cho YU, Chi HS, Lee EH, et al., (2009). Comparison of clinicopathologic findings according to JAK2 V617F mutation in patients with essential thrombocythemia. Int $\mathrm{J}$ Hematol; 89(1): 39-44".

[39] Ivanyi JL, Marton E, Plander M (2011). Significance of the JAK2V617F mutation in patients with chronic myeloproliferative neoplasia. Orv Hetil; 152(45): 1795-1803".

[40] Pósfai E, Marton I, Király PA. JAK2 V617F, MPL, and CALR Mutations in Essential Thrombocythaemia and Major Thrombotic Complications: A Single-Institute Retrospective Analysis. Pathol Oncol Res".

[41] Barbui T, Finazzi G, Carobbio A, et al., (2012). Development and validation of an International Prognostic Score of thrombosis in World Health Organization essential thrombocythemia (IPSET-thrombosis). Blood; 120(26): 51285133".

[42] Tefferi A (2013). Polycythemia vera and essential thrombocythemia: 2013 update on diagnosis, riskstratification, and management. Am J Hematol; 88(6): 507$516 "$.

[43] De Stefano V, Za T, Rossi E, et al., (2010). Increased risk of recurrent thrombosis in patients with essential thrombocythemia carrying the homozygous JAK2 V617F mutation. Ann Hematol; 89: 141-146".

[44] Musolino C, Alonci A, Bellomo G, et al., (2000). Markers of endothelial and platelet status in patients with essential thrombocythemia and polycythemia vera. Hematology; 4: 397-402".

[45] Chakrabarti S, Varghese S, Vitseva O, et al., (2005). CD40 ligand influences platelet release of reactive oxygen intermediates. Arterioscler Thromb Vasc Biol; 25: 24282434".

[46] Khan SY, Kelher MR, Heal JM, et al., (2006). Soluble CD40 ligand accumulates in stored blood components, primes neutrophils through CD40, and is a potential cofactor in the development of transfusion-related acute lung injury. Blood; 108: 2455-2462".

[47] Arellano-Rodrigo E, Alvarez-Larran A, Reverter JC, et al., (2009). Platelet turnover, coagulation factors, and soluble markers of platelet and endothelial activation in essential thrombocythemia: Relationship with thrombosis occurrence and JAK2 V617F allele burden. Am J Hematol; 84: 102-108". 